\title{
State-dependent distributed-delay model of orthogonal cutting
}

\author{
Tamás G. Molnár • Tamás Insperger • Gábor Stépán
}

Received: date / Accepted: date

\begin{abstract}
In this paper we present a model of turning operations with state-dependent distributed time delay. We apply the theory of regenerative machine tool chatter and describe the dynamics of the tool-workpiece system during cutting by delay-differential equations. We model the cutting-force as the resultant of a force system distributed along the rake face of the tool, which results in a short distributed delay in the governing equation superimposed on the large regenerative delay. According to the literature on stress distribution along the rake face, the length of the chip-tool interface, where the distributed cutting-force system is acting, is function of the chip thickness, which depends on the vibrations of the tool-workpiece system due to the regenerative effect. Therefore, the additional short delay is state-dependent. It is shown that involving statedependent delay in the model does not affect linear stability properties, but does affect the nonlinear dynamics of the cutting process. Namely, the sense of the Hopf bifurcation along the stability boundaries may turn from sub- to supercritical at certain spindle speed regions.
\end{abstract}

Keywords metal cutting · turning · delay-differential equation · distributed delay · state-dependent delay · Hopf bifurcation

\section{Introduction}

Many engineering problems can be described by delaydifferential equations (DDEs) involving distributed de-

Tamás G. Molnár, Tamás Insperger, Gábor Stépán Department of Applied Mechanics

Budapest University of Technology and Economics

E-mail: molnar@mm.bme.hu, insperger@mm.bme.hu, stepan@mm.bme.hu lays. Examples include wheel-shimmy [37,41], delaycoupled networks [40], predictive control systems [30, $16,31]$ and machine tool vibrations [36,11,27,32]. Differential equations involving state-dependent delays also often show up in different fields of science, such as classical electrodynamics [13], population models [26,35], market dynamics [6], and, again, machine tool vibrations $[23,15,3,28,29]$. In this paper, a model is presented for machining, which involves a combination of these two types of delays: a state-dependent distributed delay.

A model of regenerative machine tool vibrations is analyzed with special attention to the distribution of the contact force between the tool's rake face and the workpiece. According to experiments [24,42,44,39], the length and the shape of the force distribution depends on the chip thickness, which implies that the corresponding distributed delay in the model equation is state-dependent. The scope of this paper is to explore whether this state-dependent delay affects the linear and the global stability properties of the machining operation compared to the same model with distributed delay of constant length.

Linearization of state-dependent delay-differential equations (SD-DDEs) is complicated by the fact that the solution of the system is not differentiable with respect to the state-dependent delay (see, e.g., [20] and the references therein). Consequently, linearization in the traditional sense is not possible, but a linear DDE can be constructed, which is associated to the original SD-DDE in the sense that they have the same local stability properties. Here, we follow the linearization technique developed by Hartung and Turi in [19]. Note that there are similar methods for different classes of state-dependent delay-differential equations, see, for 

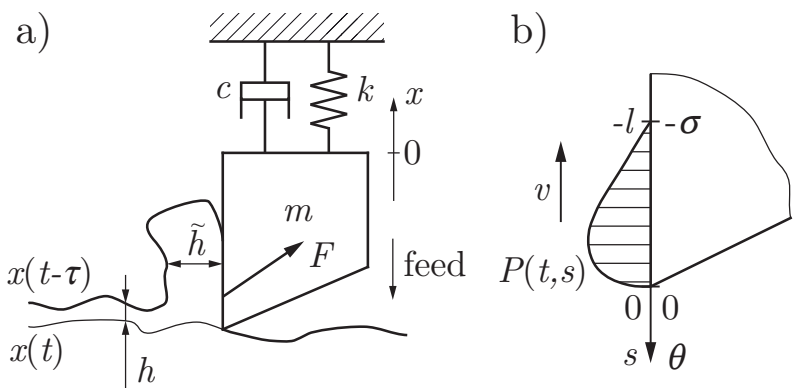

Fig. 1 Single-degree-of-freedom model of orthogonal cutting (a); cutting-force distribution along the tool's rake face (b)

instance, [10,21,17]. A detailed survey about general types of SD-DDEs is given in [18].

The outline of the paper is as follows. Section 2 briefly revises an earlier model [36] of orthogonal cutting, where distributed delays are involved in the governing equation. Section 3 extends this model by considering the state-dependency of the length of the distributed delay. The linear system associated with the state-dependent delay-differential equation is determined and compared with that of the corresponding constantlength delay model in Section 4. Numerical bifurcation analysis using DDE-BIFTOoL is performed in Section 5. Finally, conclusions are drawn in Section 6 .

\section{Distributed-delay model}

In this paper we investigate the dynamics of turning operations by analyzing the single-degree-of-freedom model of orthogonal cutting. The mechanical model is presented in Fig. 1. Given the modal mass $m$, damping $c$, and stiffness $k$ corresponding to the dominant vibration mode of the machining system, the equation of motion of the tool can be written in the form

$m \ddot{x}(t)+c \dot{x}(t)+k x(t)=F_{x}(t)$,

where $F_{x}(t)$ is the $x$-component of the cutting-force acting on the tool.

Here we model the cutting-force as the resultant of a force system acting on the tool's rake face. The cuttingforce system is assumed to be distributed along the interface between the chip and the tool. The length of the contact region is denoted by $l$ as shown in panel (b) of Fig. 1. This model was investigated by Stépán [36] assuming that the contact length $l$ and the speed $v$ by which the chip slips along the rake face is constant in time. Here, this model is extended to state-dependent contact length.

The distributed cutting-force $P(t, s)$ acting on the rake face can be described using the local spatial coor- dinate $s \in[-l, 0]$ running from the point of chip separation at $s=-l$ to the tool tip at $s=0$. If the speed $v$ is constant, then the cutting-force distribution can be written as a function of time using a local temporal coordinate $\theta=s / v, \theta \in[-\sigma, 0]$. Here $\sigma=l / v$ denotes the time it takes for a given particle of the chip to slip along the rake face of the tool, which is constant in time if the assumptions $l=$ const, $v=$ const hold. This way the cutting-force expression becomes

$F_{x}(t)=\int_{-l}^{0} P(t, s) \mathrm{d} s=\int_{-\sigma}^{0} p(t, \theta) \mathrm{d} \theta$,

where $p(t, \theta)=v P(t, v \theta)$.

From this point on, the intensity $p(t, \theta)$ distributed in the time $\theta$ is used to represent the distributed cuttingforce instead of $P(t, s)$. We assume that $p(t, \theta)$ can be decomposed multiplicatively into a time-dependent term $F_{x}^{\mathrm{T}}(t, \theta)$ and a time-independent term $w(\theta)$ :

$p(t, \theta)=F_{x}^{\mathrm{T}}(t, \theta) w(\theta), \quad \theta \in[-\sigma, 0]$.

The weight function $w(\theta)$ describes the shape of the force distribution along the tool's rake face in case of stable stationary cutting where the chip thickness is constant. The function is normalized so that it satisfies

$\int_{-\sigma}^{0} w(\theta) \mathrm{d} \theta=1$

We assume that the same weight function can be used for non-stationary cutting (chatter). In this case, the surface of the workpiece becomes wavy and the chip thickness $h(t+\theta)$ varies in time as a function of $t$ and changes along the rake face as a function of $\theta$. Therefore, the chip thickness variation affects the cutting-force, which is involved in the term $F_{x}^{\mathrm{T}}(t, \theta)$ describing the magnitude of force distribution. We assume that the magnitude $F_{x}^{\mathrm{T}}(t, \theta)$ of the cutting-force distribution can be given as a function of the chip thickness using the well-known three-quarter rule [1], namely

$F_{x}^{\mathrm{T}}(t, \theta)= \begin{cases}\operatorname{Kah}^{q}(t+\theta) & \text { if } h(t+\theta) \geq 0, \\ 0 & \text { if } h(t+\theta)<0,\end{cases}$

where $K$ is the cutting coefficient to be determined by experiments, $q=3 / 4$ is the cutting-force exponent, and $a$ is the width of cut. The case $h(t+\theta)<0$ applies when the tool loses contact with the workpiece due to largeamplitude chatter. Further on, we exclude this case and assume $h(t+\theta) \geq 0$, that is, the tool remains in cut during the entire machining operation. Note that the dependence of the cutting force $F_{x}$ on the vibration velocity $\dot{x}$ is neglected here. Therefore, this model does not describe the friction-induced stick-slip phenomenon [29]. 
According to the theory of regenerative machine tool chatter, the uncut chip thickness $h(t)$ at the tool tip depends on the actual position of the cutting tool and the position at the previous cut:

$h(t)=h_{0}+x(t-\tau)-x(t)$,

where $h_{0}$ is the prescribed uncut chip thickness, which is equal to the feed per revolution in case of orthogonal cutting. Whereas $\tau$ is the regenerative delay, which is now equal to the rotation period and can be expressed with the angular velocity $\Omega$ of the workpiece: $\tau=2 \pi / \Omega$. Experiments show $[24,39]$ that the chip thickness $\tilde{h}(t, \theta)$ along the rake face (also called the deformed chip thickness) is proportional to the shifted uncut chip thickness $h(t+\theta)$ (also called undeformed chip thickness): $\tilde{h}(t, \theta)=C h(t+\theta)$, where $1<C<10$ is a constant depending primarily on the workpiece material and the rake angle. Thus,

$\tilde{h}(t, \theta)=C\left(h_{0}+x(t-\tau+\theta)-x(t+\theta)\right), \quad \theta \in[-\sigma, 0]$.

Substituting the cutting-force expression given by Eqs. (2)-(6) back into the equation of motion (1), and dividing by the modal mass $m$, we obtain

$$
\begin{aligned}
\ddot{x}(t) & +2 \zeta \omega_{\mathrm{n}} \dot{x}(t)+\omega_{\mathrm{n}}^{2} x(t) \\
= & \frac{K a}{m} \int_{-\sigma}^{0}\left[h_{0}+x(t-\tau+\theta)-x(t+\theta)\right]^{q} w(\theta) \mathrm{d} \theta,
\end{aligned}
$$

where $\omega_{\mathrm{n}}=\sqrt{k / m}$ denotes the natural angular frequency of the undamped system and $\zeta=c /(2 \sqrt{\mathrm{km}})$ is the damping ratio. We can see that Eq. (8) is a delaydifferential equation with two distributed-delay terms over $[-\tau-\sigma,-\tau]$ and $[-\sigma, 0]$. The kernel $w(\theta)$ of the distributed-delay terms is determined by the shape of force distribution along the tool's rake face at stationary cutting.

\section{State-dependent delay model}

Equation (8) has already been derived by Stépán [36]. Now we improve his model by recognizing that the size $l$ of the chip-tool interface is not constant, but is a function of the time-varying uncut chip thickness $h$. According to experimental results presented in $[24,42,44,39]$, the relation between the contact length and chip thickness can be described using a linear (or shifted linear) function in a wide range of uncut chip thickness values. Therefore, we assume that the contact length is proportional to the uncut chip thickness at the tool tip:

$l(t)=A h(t)=A\left(h_{0}+x(t-\tau)-x(t)\right)$.
Since the cutting experiments are usually performed under chatter-free conditions, the measured constant $A$ is actually the ratio of the stationary contact length $l_{0}$ and the prescribed chip thickness $h_{0}$. According to measurements reported in $[24,42,44,39]$, this ratio is in the range $2<A<20$ depending on the workpiece material (e.g. $A \approx 5$ for steel, $A \approx 10$ for copper in [42], and $A \approx 20$ for aluminum in [24]).

Based on Eq. (9), the contact length $l$ depends on the position of the tool. Thus, we will emphasize the state-dependency by the notation $l=l\left(x_{t}\right)$, where the function $x_{t}(\vartheta)=x(t+\vartheta)$ represents the tool's position over the delay period $\vartheta \in[-\rho, 0]$, and $\rho$ is the maximum delay that can occur in the system.

Furthermore, we keep the assumption that the chip slips along the rake face by a constant speed $v$. From Eq. (9) it follows that the additional short delay is also state-dependent:

$\sigma\left(x_{t}\right)=\frac{l\left(x_{t}\right)}{v}=\frac{A}{v}\left(h_{0}+x(t-\tau)-x(t)\right)$.

Note that in [36] it was assumed that the short delay $\sigma$ is proportional to the regenerative delay $\tau: \sigma=\varepsilon \tau$, where $\varepsilon$ is a small constant value. If we simplify Eq. (10) by assuming stationary cutting with constant contact length $l_{0}=A h_{0}$ and assuming that $v$ is equal to the constant cutting speed, $v=\Omega D / 2=D \pi / \tau$, then we get the same relation $\sigma=\varepsilon \tau$ with $\varepsilon=A h_{0} / D \pi$. Here $D$ denotes the tool diameter, hence $\varepsilon$ is equivalent to the ratio of the contact length $l_{0}=A h_{0}$ and the workpiece perimeter $D \pi$. Typical values of this ratio are in the range $0.0005<\varepsilon<0.05[5,7]$. However, in case of the state-dependent short delay described by Eq. (10), the ratio $\sigma\left(x_{t}\right) / \tau=\varepsilon\left(x_{t}\right)$ is no longer constant but statedependent.

Since the delay $\sigma\left(x_{t}\right)$ varies according to the tool position, the domain $\left[-\sigma\left(x_{t}\right), 0\right]$ and the magnitude of the kernel function $w(\theta)$ also changes. Therefore, the kernel function can be written as

$$
w(\theta)=\frac{1}{\sigma\left(x_{t}\right)} f\left(\frac{\theta}{\sigma\left(x_{t}\right)}\right),
$$

where $f$ is a function, which characterizes the shape of the stationary force distribution along the tool's rake face independently of the state $x_{t}$. It is reasonable to introduce a coordinate $\hat{\theta}=\theta / \sigma\left(x_{t}\right) \in[-1,0]$, which is interpreted on a fixed domain independently of $x_{t}$. Due to Eq. (4), the function $f$ has the property

$$
\int_{-1}^{0} f(\hat{\theta}) \mathrm{d} \hat{\theta}=1 .
$$

The shape $f(\hat{\theta})$ of the force distribution can be determined based on the results of stress distribution measurements along the tool's rake face. Summaries of the 
a)
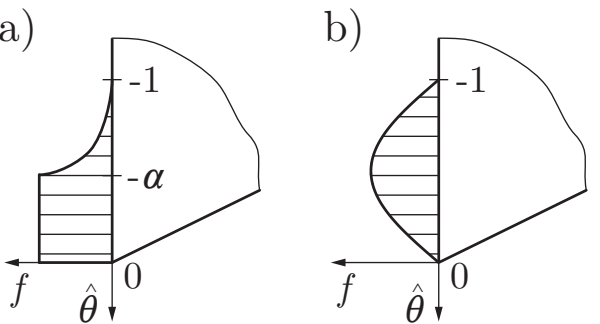

Fig. 2 The shape of force distribution along the cutting-tool

experimental results can be found in $[45,2,25]$. In case of zero rake angle, the $x$-directional component of the stresses on the rake face is the shear stress. According to the literature on shear stress distribution measurements, two different types of shear stress distributions were identified. Several measurements $[43,8,24,5,9,7]$ showed that the shear stress has a plateau near the tool tip along a certain sticking length $l_{\mathrm{s}}$, and then decays to zero at the point of chip separation. The corresponding shape function $f(\hat{\theta})$ can be approximated using a constant and an exponential function as shown in panel (a) of Fig. 2, and can be written in the form

$f(\hat{\theta})= \begin{cases}\frac{1-\mathrm{e}^{-\alpha+1}}{2-(\alpha+1) \mathrm{e}^{-\alpha+1}} & \text { if } \hat{\theta} \in[-\alpha, 0], \\ \frac{1-\mathrm{e}^{\hat{\theta}+1}}{2-(\alpha+1) \mathrm{e}^{-\alpha+1}} & \text { if } \hat{\theta} \in[-1,-\alpha),\end{cases}$

where $\alpha=l_{\mathrm{s}} / l$ denotes the sticking length to contact length ratio. According to other shear stress distribution measurements $[8,4]$, the shear stress initiates from a small value at the tip, reaches a maximum in the middle of the contact region, and then decays to zero. We can approximate this function by a half-sine wave:

$f(\hat{\theta})=-\frac{\pi}{2} \sin (\pi \hat{\theta})$,

which is also shown in panel (b) of Fig. 2 .

Eq. (11) shows the form of the kernel $w(\theta)$, which can be substituted back into Eq. (8). The resulting equation of motion becomes

$$
\begin{aligned}
& \ddot{x}(t)+2 \zeta \omega_{\mathrm{n}} \dot{x}(t)+\omega_{\mathrm{n}}^{2} x(t) \\
& =\frac{K a}{m} \int_{-\sigma\left(x_{t}\right)}^{0}\left[h_{0}+x(t-\right. \\
& \quad \times \frac{1}{\sigma\left(x_{t}\right)} f\left(\frac{\theta}{\sigma\left(x_{t}\right)}\right) \mathrm{d} \theta,
\end{aligned}
$$

where $\sigma\left(x_{t}\right)$ must be substituted from Eq. (10). The governing equation (15) is a delay-differential equation containing two state-dependent distributed-delay terms.
Note that the state-dependent short delay $\sigma\left(x_{t}\right)$ appears in the governing equation (15) explicitly. Scaling the integral term using $\hat{\theta}=\theta / \sigma\left(x_{t}\right)$, one obtains

$$
\begin{aligned}
\ddot{x}(t)+2 \zeta \omega_{\mathrm{n}} \dot{x}(t)+\omega_{\mathrm{n}}^{2} x(t) & \\
=\frac{K a}{m} \int_{-1}^{0}\left[h_{0}+x\left(t-\tau+\hat{\theta} \sigma\left(x_{t}\right)\right)-\right. & \left.x\left(t+\hat{\theta} \sigma\left(x_{t}\right)\right)\right]^{q} \\
& \times f(\hat{\theta}) \mathrm{d} \hat{\theta},
\end{aligned}
$$

which does not contain the term $\sigma\left(x_{t}\right)$ explicitly any more. Note that state-dependent delay differential equations are always nonlinear, since the state appears in its own argument. Thus, Eqs. (15) and (16) would still be nonlinear even if the cutting exponent was $q=1$. In the next section, we derive the linear delay-differential equation associated with Eq. (15).

\section{Associated linear system}

The associated linear system, whose local stability properties are equivalent to the original state-dependent delaydifferential equation, is determined using the method published in [19]. For this purpose, we write Eq. (15) in the form investigated in [19]. First, we introduce the coordinate $\hat{x}(t)$ shifted to the equilibrium $\bar{x}$ of Eq. (15):

$x(t)=\bar{x}+\hat{x}(t), \quad \bar{x}=\frac{K a h_{0}^{q}}{m \omega_{\mathrm{n}}^{2}}$.

Accordingly, Eq. (15) can be written in the form

$$
\begin{aligned}
\ddot{\hat{x}}(t)+2 \zeta \omega_{\mathrm{n}} \dot{\hat{x}}(t)+\omega_{\mathrm{n}}^{2} \hat{x}(t) & \\
=\frac{K a}{m} \int_{-\sigma\left(\hat{x}_{t}\right)}^{0}\left[\left(h_{0}+\hat{x}(t\right.\right. & \left.-\tau+\theta)-\hat{x}(t+\theta))^{q}-h_{0}^{q}\right] \\
& \times \frac{1}{\sigma\left(\hat{x}_{t}\right)} f\left(\frac{\theta}{\sigma\left(\hat{x}_{t}\right)}\right) \mathrm{d} \theta,
\end{aligned}
$$

where property (12) of $f$ was taken into account when moving $h_{0}^{q}$ inside the integral. The equilibrium of Eq. (18) is therefore $\hat{x}(t) \equiv 0$.

Then, we replace the state-dependent lower limit of the integration by a constant using the Heaviside step function $H$ in the kernel of the distributed-delay term:

$$
\begin{aligned}
& \ddot{\hat{x}}(t)+2 \zeta \omega_{\mathrm{n}} \dot{\hat{x}}(t)+\omega_{\mathrm{n}}^{2} \hat{x}(t) \\
& =\frac{K a}{m} \int_{-\sigma_{\max }}^{0}\left[\left[h_{0}+\hat{x}(t-\tau+\theta)-\hat{x}(t+\theta)\right]^{q}-h_{0}^{q}\right] \\
& \quad \times \frac{1}{\sigma\left(\hat{x}_{t}\right)} f\left(\frac{\theta}{\sigma\left(\hat{x}_{t}\right)}\right) H\left(\theta+\sigma\left(\hat{x}_{t}\right)\right) \mathrm{d} \theta
\end{aligned}
$$

where $\sigma_{\max }=\rho-\tau$ is the maximal length of the additional short delay. Finally, we expand the term $\left[h_{0}+\right.$ 
$\hat{x}(t-\tau+\theta)-\hat{x}(t+\theta)]^{q}$ into Taylor series around $h_{0}$ and obtain

$$
\begin{aligned}
& \ddot{\hat{x}}(t)+2 \zeta \omega_{\mathrm{n}} \dot{\hat{x}}(t)+\omega_{\mathrm{n}}^{2} \hat{x}(t) \\
& =\frac{K a}{m} \int_{-\sigma_{\max }}^{0}[\hat{x}(t-\tau+\theta)-\hat{x}(t+\theta)] \\
& \times\left[q h_{0}^{q-1}+\frac{1}{2} q(q-1) h_{0}^{q-2}[\hat{x}(t-\tau+\theta)-\hat{x}(t+\theta)]+\text { h.o.t. }\right] \\
& \quad \times \frac{1}{\sigma\left(\hat{x}_{t}\right)} f\left(\frac{\theta}{\sigma\left(\hat{x}_{t}\right)}\right) H\left(\theta+\sigma\left(\hat{x}_{t}\right)\right) \mathrm{d} \theta,
\end{aligned}
$$

where h.o.t. stands for higher-order terms.

In [19], a method was proposed to determine the equivalent linear system for a class of state-dependent delay-differential equations of form

$$
\begin{aligned}
\dot{\hat{\mathbf{z}}}(t) & =\mathbf{g}\left(\hat{\mathbf{z}}(t), \Lambda\left(\hat{\mathbf{z}}_{t}, \hat{\mathbf{z}}_{t}\right)\right), \\
\Lambda(\boldsymbol{\psi}, \boldsymbol{\phi}) & =\int_{-\sigma_{\max }}^{0} \mathrm{~d}_{\theta} \boldsymbol{\mu}(\theta, \boldsymbol{\psi}) \boldsymbol{\phi}(\theta),
\end{aligned}
$$

where $\mathbf{g}$ is a continuously differentiable function satisfying $\mathbf{g}(\mathbf{0}, 0)=\mathbf{0}$ and $\boldsymbol{\mu}(., \boldsymbol{\psi})$ is of bounded variation (see [19] for more details). Equation (20) can be represented in form (21) by introducing

$$
\begin{aligned}
& \hat{\mathbf{z}}(t)=\left[\begin{array}{c}
\hat{x}(t) \\
\dot{\hat{x}}(t)
\end{array}\right], \quad \hat{\mathbf{z}}_{t}(\vartheta)=\hat{\mathbf{z}}(t+\vartheta), \quad \vartheta \in[-\rho, 0], \\
& \mathbf{g}(\mathbf{U}, V)=\left[\begin{array}{c}
U_{2} \\
-2 \zeta \omega_{\mathrm{n}} U_{2}-\omega_{\mathrm{n}}^{2} U_{1}+\frac{K a}{m} V
\end{array}\right], \\
& \Lambda(\boldsymbol{\psi}, \boldsymbol{\phi})=\int_{-\sigma_{\max }}^{0}\left[\phi_{1}(-\tau+\theta)-\phi_{1}(\theta)\right] \\
& \times\left[q h_{0}^{q-1}+\frac{1}{2} q(q-1) h_{0}^{q-2}\left[\psi_{1}(-\tau+\theta)-\psi_{1}(\theta)\right]+\text { h.o.t. }\right] \\
& \times \frac{1}{\sigma(\boldsymbol{\psi})} f\left(\frac{\theta}{\sigma(\boldsymbol{\psi})}\right) H(\theta+\sigma(\boldsymbol{\psi})) \mathrm{d} \theta, \\
& \sigma(\boldsymbol{\psi})=\frac{A}{v}\left[h_{0}+\psi_{1}(-\tau)-\psi_{1}(0)\right] \text {. }
\end{aligned}
$$

Here, $U_{1}, U_{2}, \phi_{1}$, and $\psi_{1}$ are the corresponding elements of vectors $\mathbf{U}, \phi$, and $\boldsymbol{\psi}$, respectively.

According to [19], the linear system associated with Eq. (21) can be written in the form

$$
\dot{\mathbf{u}}(t)=\mathrm{D}_{1} \mathbf{g}(\mathbf{0}, 0) \mathbf{u}(t)+\mathrm{D}_{2} \mathbf{g}(\mathbf{0}, 0) \Lambda\left(\mathbf{0}, \mathbf{u}_{t}\right),
$$

where $\mathrm{D}_{i}$ denotes the derivative with respect to the $i$ th argument $(i=1,2)$, and $\mathbf{u}(t)=[\xi(t) \dot{\xi}(t)]^{\mathrm{T}}$ stands for the perturbation around the trivial solution $\hat{\mathbf{z}}(t) \equiv \mathbf{0}$. The derivatives of $\mathbf{g}$ obtained from Eq. (23) are

$$
\mathrm{D}_{1} \mathbf{g}(\mathbf{0}, 0)=\left[\begin{array}{cc}
0 & 1 \\
-\omega_{\mathrm{n}}^{2} & -2 \zeta \omega_{\mathrm{n}}
\end{array}\right], \quad \mathrm{D}_{2} \mathbf{g}(\mathbf{0}, 0)=\left[\begin{array}{c}
0 \\
\frac{K a}{m}
\end{array}\right]
$$

Whereas from Eq. (24) we get

$$
\begin{aligned}
\Lambda(\mathbf{0}, \boldsymbol{\phi})=\int_{-\sigma_{\max }}^{0} & \left.\phi_{1}(-\tau+\theta)-\phi_{1}(\theta)\right] \\
& \times q h_{0}^{q-1} \frac{1}{\bar{\sigma}} f\left(\frac{\theta}{\bar{\sigma}}\right) H(\theta+\bar{\sigma}) \mathrm{d} \theta,
\end{aligned}
$$

where $\bar{\sigma}$ denotes the constant solution for the short delay obtained from Eq. (25):

$\bar{\sigma}=\frac{A h_{0}}{v}$.

Substitution of Eqs. (27) and (28) into Eq. (26) gives

$\frac{\mathrm{d}}{\mathrm{d} t}\left[\begin{array}{c}\xi(t) \\ \dot{\xi}(t)\end{array}\right]=\left[\begin{array}{c}\dot{\xi}(t) \\ -\omega_{\mathrm{n}}^{2} \xi(t)-2 \zeta \omega_{\mathrm{n}} \dot{\xi}(t)+\frac{K a q h_{0}^{q-1}}{m} I\left(\xi_{t}\right)\end{array}\right]$

with

$I\left(\xi_{t}\right)=\int_{-\bar{\sigma}}^{0}[\xi(t-\tau+\theta)-\xi(t+\theta)] \frac{1}{\bar{\sigma}} f\left(\frac{\theta}{\bar{\sigma}}\right) \mathrm{d} \theta$,

which is equivalent to the second-order linear system

$$
\begin{aligned}
& \ddot{\xi}(t)+2 \zeta \omega_{\mathrm{n}} \dot{\xi}(t)+\omega_{\mathrm{n}}^{2} \xi(t) \\
& =\frac{K a q h_{0}^{q-1}}{m} \int_{-\bar{\sigma}}^{0}[\xi(t-\tau+\theta)-\xi(t+\theta)] \frac{1}{\bar{\sigma}} f\left(\frac{\theta}{\bar{\sigma}}\right) \mathrm{d} \theta .
\end{aligned}
$$

It can be seen that Eq. (32) associated with the statedependent delay-differential equation (15) is the same as the linear part of model (8) with constant delay $\sigma=$ $\bar{\sigma}$. Thus the state-dependency has no effect on the linear stability of the machining operation, and the stability charts derived in [36] are valid without any change for the extended state-dependent delay model, too.

Note that the same linear system can also be obtained by formal differentiations from Eq. (16), which has a nontrivial equilibrium $x(t) \equiv \bar{x}$. In order to show this, Eq. (16) is represented in first-order form:

$\dot{\mathbf{z}}(t)=\int_{-1}^{0} \mathbf{g}\left(\mathbf{z}(t), \eta\left(\mathbf{z}_{t}\right)\right) f(\hat{\theta}) \mathrm{d} \hat{\theta}$

where

$\mathbf{z}(t)=\left[\begin{array}{c}x(t) \\ \dot{x}(t)\end{array}\right], \quad \mathbf{z}_{t}(\vartheta)=\mathbf{z}(t+\vartheta), \quad \vartheta \in[-\rho, 0]$,

$\mathbf{g}\left(\mathbf{z}(t), \eta\left(\mathbf{z}_{t}\right)\right)=\left[\begin{array}{c}\dot{x}(t) \\ -2 \zeta \omega_{\mathrm{n}} \dot{x}(t)-\omega_{\mathrm{n}}^{2} x(t)+\frac{K a}{m} \eta^{q}\left(x_{t}\right)\end{array}\right]$,

$\eta\left(x_{t}\right)=h_{0}+x\left(t-\tau+\hat{\theta} \sigma\left(x_{t}\right)\right)-x\left(t+\hat{\theta} \sigma\left(x_{t}\right)\right)$. 
Note that $\eta\left(\mathbf{z}_{t}\right)=\eta\left(x_{t}\right)$ is linear in $x_{t}$. In Eq. (33), we took advantage of property (12) of $f$ when taking the terms $\dot{x}(t),-2 \zeta \omega_{\mathrm{n}} \dot{x}(t)$, and $-\omega_{\mathrm{n}}^{2} x(t)$ inside the integral.

The associated linear system around the constant solution $\mathbf{z}(t) \equiv \overline{\mathbf{z}}=\left[\begin{array}{ll}\bar{x} & 0\end{array}\right]^{\mathrm{T}}$ of Eq. (33) can be written in the form

$$
\begin{aligned}
\dot{\mathbf{u}}(t)=\int_{-1}^{0} & \mathrm{D}_{1} \mathbf{g}\left(\overline{\mathbf{z}}, \eta\left(\overline{\mathbf{z}}_{t}\right)\right) \mathbf{u}(t) f(\hat{\theta}) \mathrm{d} \hat{\theta} \\
& +\int_{-1}^{0} \mathrm{D}_{2} \mathbf{g}\left(\overline{\mathbf{z}}, \eta\left(\overline{\mathbf{z}}_{t}\right)\right) \operatorname{D} \eta\left(\overline{\mathbf{z}}_{t}\right) \mathbf{u}_{t} f(\hat{\theta}) \mathrm{d} \hat{\theta}
\end{aligned}
$$

Substituting $x(t) \equiv \bar{x}$ into Eq. (10) gives the constant solution (29) for the short delay. Calculation of the derivatives in Eq. (37) results in

$$
\begin{aligned}
& \mathrm{D}_{1} \mathbf{g}\left(\overline{\mathbf{z}}, \eta\left(\overline{\mathbf{z}}_{t}\right)\right)=\left[\begin{array}{cc}
0 & 1 \\
-\omega_{\mathrm{n}}^{2}-2 \zeta \omega_{\mathrm{n}}
\end{array}\right], \\
& \mathrm{D}_{2} \mathbf{g}\left(\overline{\mathbf{z}}, \eta\left(\overline{\mathbf{z}}_{t}\right)\right)=\left[\begin{array}{c}
0 \\
\frac{K a q h_{0}^{q-1}}{m}
\end{array}\right] .
\end{aligned}
$$

The term $\mathrm{D} \eta\left(\overline{\mathbf{z}}_{t}\right) \mathbf{u}_{t}$ can be written as

$$
\begin{aligned}
\mathrm{D} \eta\left(\overline{\mathbf{z}}_{t}\right) \mathbf{u}_{t}=\left[\mathrm{D}_{x_{t}} \eta\left(\overline{\mathbf{z}}_{t}\right)\right. & \left.\mathrm{D}_{\dot{x}_{t}} \eta\left(\overline{\mathbf{z}}_{t}\right)\right]\left[\begin{array}{c}
\xi_{t} \\
\dot{\xi}_{t}
\end{array}\right] \\
= & \mathrm{D}_{x_{t}} \eta\left(\overline{\mathbf{z}}_{t}\right) \xi_{t}+\mathrm{D}_{\dot{x}_{t}} \eta\left(\overline{\mathbf{z}}_{t}\right) \dot{\xi}_{t},
\end{aligned}
$$

where $\mathrm{D}_{x_{t}} \eta$ and $\mathrm{D}_{\dot{x}_{t}} \eta$ denote the Frechét derivatives of $\eta$ with respect to $x_{t}$ and $\dot{x}_{t}$, respectively. Taking the Frechét derivative of both sides of Eq. (36) with respect to $x_{t}$ and $\dot{x}_{t}$ gives

$$
\begin{aligned}
& \mathrm{D}_{x_{t}} \eta\left(\overline{\mathbf{z}}_{t}\right) \xi_{t}=\xi(t-\tau+\hat{\theta} \bar{\sigma})-\xi(t+\hat{\theta} \bar{\sigma}), \\
& \mathrm{D}_{\dot{x}_{t}} \eta\left(\overline{\mathbf{z}}_{t}\right) \dot{\xi}_{t}=0 .
\end{aligned}
$$

Substitution of Eqs. (38)-(40) into Eq. (37) and scaling the integral by $\theta=\bar{\sigma} \hat{\theta}$ give Eq. (30).

\section{Numerical analysis of the nonlinear system}

Although state-dependency does not affect the stability of small-amplitude vibrations, it modifies the nonlinear behavior and affects large-amplitude chatter. In some cases, relevant qualitative changes can occur in the dynamics of the nonlinear system due to the statedependent delay. For instance, it was shown in [22] for a two-degrees-of-freedom model of turning operations that the state-dependency of the regenerative delay can change the sense of the Hopf bifurcation: at some segments of the linear stability boundaries, the Hopf bifurcation turns from sub- to supercritical.

We investigate the effect of state-dependency in the current model by analyzing Eq. (16) using DDE-BIFTooL
$[14,34]$. In order to accommodate the system to DDEBiftool, we need to approximate Eq. (16), and transform it into a more convenient form.

First, we reduce the number of parameters by introducing the dimensionless time $\tilde{t}=\omega_{\mathrm{n}} t$, the dimensionless delays $\tilde{\tau}=\omega_{\mathrm{n}} \tau$ and $\tilde{\sigma}\left(x_{t}\right)=\omega_{\mathrm{n}} \sigma\left(x_{t}\right)$, the dimensionless position $\tilde{x}=x / h_{0}$, and the dimensionless chip width $p=K a q h_{0}^{q-1} /\left(m \omega_{\mathrm{n}}^{2}\right)$. After dropping the tilde, Eq. (16) can be represented in dimensionless form as

$$
\begin{aligned}
x^{\prime \prime}(t)+2 \zeta x^{\prime}(t)+x(t) & \\
=\frac{p}{q} \int_{-1}^{0}\left[1+x\left(t-\tau+\hat{\theta} \sigma\left(x_{t}\right)\right)-x(\right. & \left.\left.+\hat{\theta} \sigma\left(x_{t}\right)\right)\right]^{q} \\
& \times f(\hat{\theta}) \mathrm{d} \hat{\theta} .
\end{aligned}
$$

Note that for small spindle speeds when $\Omega$ approaches zero, the delays become infinitely large as $\tau \rightarrow \infty$. In order to avoid a badly-scaled system caused by very large delays, we rescale time as $T=t / \tau$, which yields

$$
\begin{aligned}
& \\
x^{\prime \prime}(T)+2 \zeta \tau x^{\prime}(T)+\tau^{2} x(T) & \\
=\frac{p \tau^{2}}{q} \int_{-1}^{0}\left[1+x\left(T-1+\hat{\theta} \varepsilon\left(x_{T}\right)\right)-\right. & \left.x\left(T+\hat{\theta} \varepsilon\left(x_{T}\right)\right)\right]^{q} \\
& \times f(\hat{\theta}) \mathrm{d} \hat{\theta}, \quad(42)
\end{aligned}
$$

where

$\varepsilon\left(x_{T}\right)=\frac{\bar{\sigma}}{\tau}(1+x(T-1)-x(T))$.

Furthermore, since DDE-BIFTOOL is developed for equations with point delays, we approximate the distributeddelay term in Eq. (42) by a sum of $s$ point delays as

$$
\begin{aligned}
& x^{\prime \prime}(T)+2 \zeta \tau x^{\prime}(T)+\tau^{2} x(T) \\
& \approx \frac{p \tau^{2}}{q} \sum_{k=1}^{s}\left[1+x\left(T-1-\hat{\theta}_{k} \varepsilon\left(x_{T}\right)\right)-x\left(T-\hat{\theta}_{k} \varepsilon\left(x_{T}\right)\right)\right]^{q} f_{k},
\end{aligned}
$$

where

$\hat{\theta}_{k}=\left(k-\frac{1}{2}\right) \frac{1}{s}, \quad f_{k}=\int_{-k / s}^{-(k-1) / s} f(\hat{\theta}) \mathrm{d} \hat{\theta}$.

Finally, we expand the $q$ th power of the bracketed term in Eq. (44) into a Taylor series around 1 up to third order in order to avoid the infinite derivative at zero (since $q<1)$. Introducing the shifted coordinate $\hat{x}(T)=x(T)-$ 


$$
\begin{aligned}
& p / q, \text { we get } \\
& \hat{x}^{\prime \prime}(T)+2 \zeta \tau \hat{x}^{\prime}(T)+\tau^{2} \hat{x}(T) \\
& \approx p \tau^{2} \sum_{k=1}^{s}\left\{\left[\hat{x}\left(T-1-\hat{\theta}_{k} \varepsilon\left(x_{T}\right)\right)-\hat{x}\left(T-\hat{\theta}_{k} \varepsilon\left(x_{T}\right)\right)\right]\right. \\
& +\eta_{2}\left[\hat{x}\left(T-1-\hat{\theta}_{k} \varepsilon\left(x_{T}\right)\right)-\hat{x}\left(T-\hat{\theta}_{k} \varepsilon\left(x_{T}\right)\right)\right]^{2} \\
& \left.+\eta_{3}\left[\hat{x}\left(T-1-\hat{\theta}_{k} \varepsilon\left(x_{T}\right)\right)-\hat{x}\left(T-\hat{\theta}_{k} \varepsilon\left(x_{T}\right)\right)\right]^{3}\right\} f_{k},
\end{aligned}
$$

where $\eta_{2}=(q-1) / 2, \eta_{3}=(q-1)(q-2) / 6$.

Equation (46) can directly be used as input for DDEBifTool. First, numerical continuation is used to determine the local stability boundaries of the system, where Hopf bifurcation occurs. Then, at each Hopf bifurcation point, the amplitude of the arising periodic orbit is computed and continued until the periodic oscillations get so large that loss of contact $(h(t)<0)$ occurs and Eq. (16) is not valid anymore.

The results are summarized in Fig. 3. Panels (a) and (b) present the stability charts of the system in the plane $(\Omega, p)$ for high and low spindle speed regions, respectively. These diagrams were calculated using the plateau-and-decay force distribution (13) with $\alpha=0.4$, assuming a damping ratio $\zeta=0.02$, a delay ratio $\bar{\sigma} / \tau=0.05$, and a cutting exponent $q=0.75$. The distributed-delay term is approximated by $s=10$ point delays. Solid line indicates the local stability boundaries of the equilibrium, where Hopf bifurcation occurs. This line coincides with the linear stability boundaries obtained analytically in [36] for the corresponding constant-delay model (8), hence the numerical analysis verifies the derivation of the associated linear system in Section 4.

Panels (c)-(g) of Fig. 3 present the bifurcation diagrams corresponding to the 2nd, 3rd, 4th, 18th, and 24th stability lobes indicated by arrows in panels (a) and (b). Branches of periodic solutions were computed at discrete points along the stability lobes. The order of the points on the stability charts and the respective order of the bifurcation curves are indicated by arrows. In the bifurcation diagrams we plot the amplitude $r=(\max \hat{x}(t)-\min \hat{x}(t)) / 2$ of periodic orbits born at the Hopf bifurcation as a function of the bifurcation parameter, the dimensionless chip width, $p$. In order to see the tendency of the branches, the amplitude $r$ is normalized by the critical amplitude $r_{\mathrm{cr}}$, which denotes the smallest amplitude where the tool first loses contact with the workpiece due to the periodic oscillations. Similarly, the bifurcation parameter $p$ is normalized by its value $p_{\text {st }}$ at the Hopf bifurcation. The dimensionless chip width corresponding to $r_{\mathrm{cr}}$ is denoted by $p_{\mathrm{cr}}$ (see panel (h) of the figure). Hence, in the normalized plane $\left(p / p_{\mathrm{st}}, r / r_{\mathrm{cr}}\right)$ each branch starts from point $(1,0)$ and ends at point $\left(p_{\text {cr }} / p_{\text {st }}, 1\right)$.

Note that the Hopf bifurcation is subcritical when a bifurcation curve starts bending to the left, and supercritical when it bends to the right. It can be concluded from panels (c)-(g) of Fig. 3 that the Hopf bifurcation changes criticality as the spindle speed $\Omega$ is decreased. In panels (a) and (b) of Fig. 3, the subcritical segment of the stability lobes is shown by a thin line, whereas thick lines indicate supercriticality. The points where the criticality turns are marked by large dots. Panel (c) shows that the bifurcation is subcritical in the highspeed region along the 2nd lobe. Then, the sense of the bifurcation turns from sub- to supercritical at the 3rd lobe, and remains supercritical down to the 14th lobe (in this particular example). At low spindle speeds, from the 15th lobe, subcritical segments appear on the right side of the stability lobes. As the spindle speed is decreased, the subcritical segments grow, and from the 24 th lobe, the bifurcation is subcritical again.

This is an important qualitative difference from the behavior of the constant-delay model (8) where the bifurcation is subcritical independently of the spindle speed, and $p_{\text {cr }} \leq p \leq p_{\text {st }}$ holds for the branches of periodic solutions [33]. In the constant-delay model, consequently, there exists a bistable region at $p_{\mathrm{cr}}<p<$ $p_{\text {st }}$ where stable stationary cutting and large-amplitude chatter coexist and the system becomes unstable to large enough perturbations. It was shown in [33] that the bistable region occupies approximately $4 \%$ of the linearly stable region for all spindle speeds. For the state-dependent model presented here, supercritical bifurcations also occur. Furthermore, at certain spindle speeds, the supercritical branches turn back to the left as shown in panel (h) of Fig. 3. Therefore, a bistable region can still exist for a supercritical Hopf bifurcation: it occupies $p_{\text {bist }}<p<p_{\text {st }}$ where $p_{\text {bist }}$ is the leftmost point of the bifurcation curve, see panel (h). The bistable boundary $p_{\text {bist }}$ is indicated by dashed line in the stability charts in panels (a) and (b) of Fig. 3. The bistable region itself is denoted by dark grey shading, whereas the globally stable region is indicated by light grey. As shown in panel (b), the bistable region grows as the spindle speed is decreased. In this sense, the gain in the linear stability boundaries at low spindle speeds is reduced by nonlinear effects.

\section{Conclusions}

In this paper, we proposed a dynamical model of turning operations where the cutting force is modeled as 


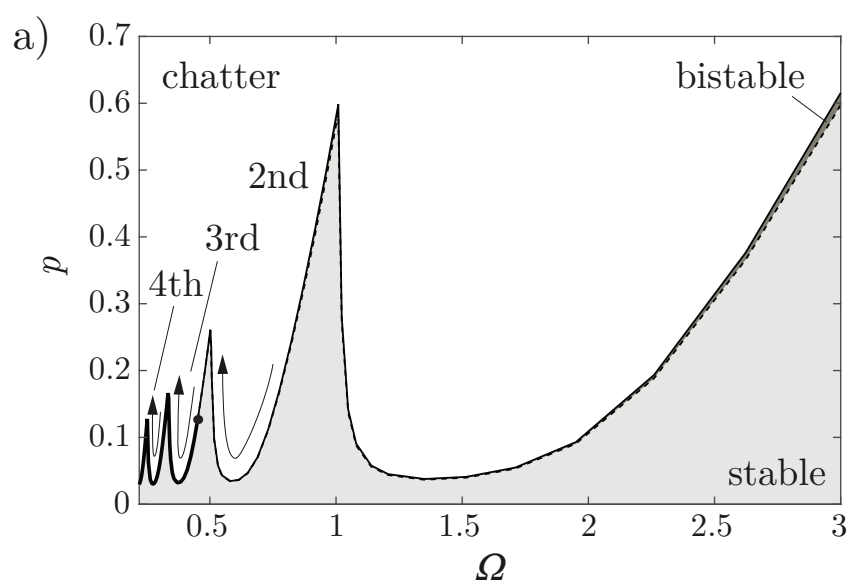

b)

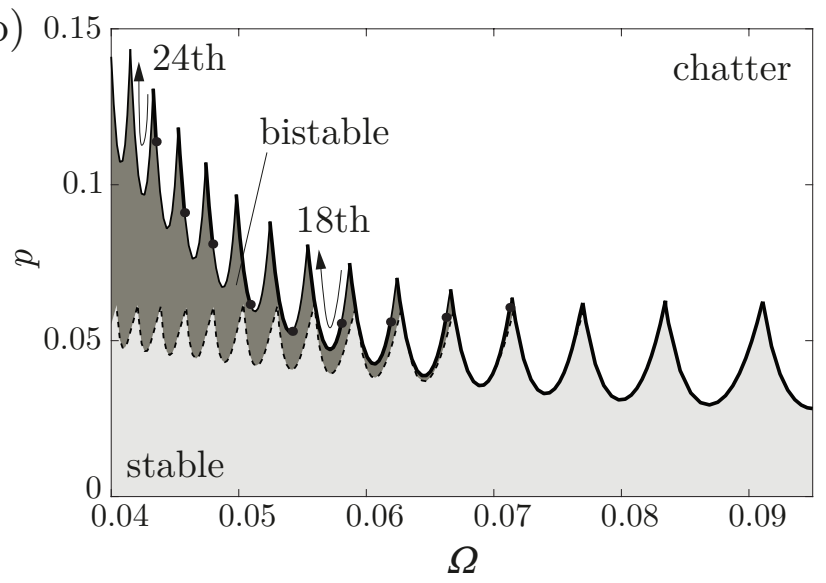

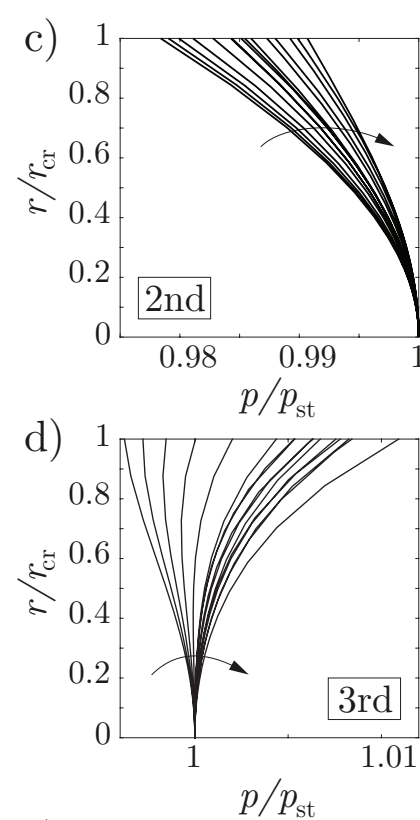

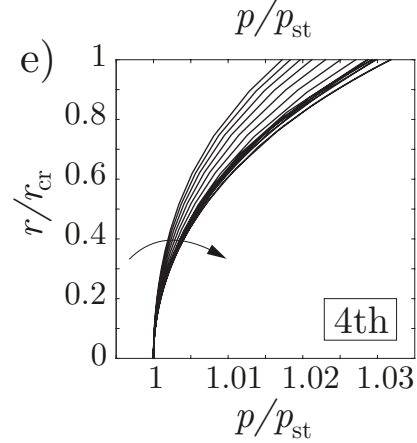

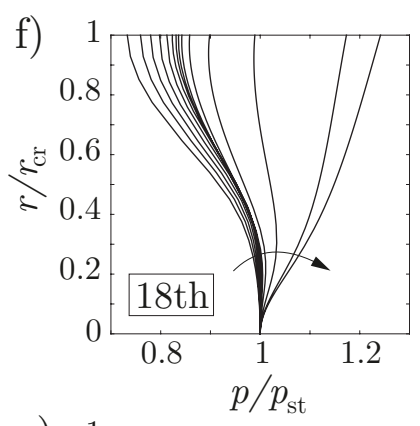

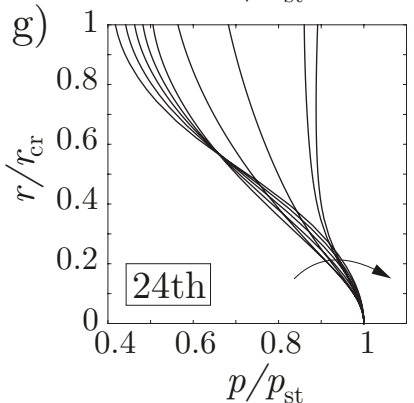

h)

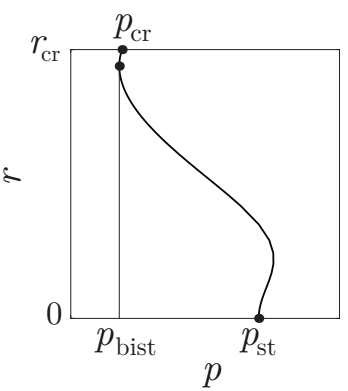

Fig. 3 Stability charts of system (16) for high (a) and low (b) spindle speed regions; bifurcation diagrams showing the amplitude of periodic orbits along the stability lobes (c)-(g); a possible bifurcation scenario (h)

a force system distributed along the chip-tool interface. We described machine tool chatter using delaydifferential equations with a short distributed delay superimposed on the large regenerative point delay. As the size of the chip-tool interface varies according to the tool position, the distributed delay becomes statedependent. Using the algorithm introduced in [19], we determined the linear system which is associated with state-dependent equation in terms of same local stability properties. We have shown that the linear system is equivalent to that of the model with constant delay. Thus, state-dependency of the short delay does not affect the linear stability properties of the cutting process.

The proposed model is qualitatively different form the one presented in [23], where a two-degrees-of-freedom turning model associated with state-dependent point delay was investigated. In the model in [23], it was shown that the stability boundaries of the associated linear system differ slightly from those of the traditional model with state-independent (constant) time- delay. The reason of the difference was that the statedependent delay appeared explicitly in the nonlinear equation, which resulted in an additional term in the associated linear system. In the model proposed in this paper, the state-dependent delay appears only in the argument of the state variables, therefore this additional term does not show up.

Although state-dependency has no effect on the local stability for the presented model of machining, it affects the nonlinear behavior. Namely, it changes the sense of the Hopf bifurcation at the stability boundaries. The bifurcation turns from sub- to supercritical at certain spindle speeds.

From engineering point of view, the subcritical Hopf bifurcation is more dangerous, since large-amplitude vibrations can evolve in certain (linearly stable) parameter regions for large enough perturbations (see $[38,12]$ ). Therefore, it is important to avoid these parameter regions and rather operate the cutting-process near the supercritical part of the Hopf boundaries. From this point of view, state-dependent delay models become 
relevant in finding the supercritical segments of the stability boundaries.

Acknowledgements This work was supported by the Hungarian National Science Foundation under grant OTKAK105433 and OTKA-K101714. The research leading to these results has received funding from the European Research Council under the European Union's Seventh Framework Programme (FP/2007-2013) / ERC Advanced Grant Agreement n. 340889.

\section{References}

1. Altintas, Y.: Manufacturing Automation - Metal Cutting Mechanics, Machine Tool Vibrations and CNC Design, Second Edition. Cambridge University Press, Cambridge (2012)

2. Astakhov, V.P., Outeiro, J.C.: Modeling of the contact stress distribution at the tool-chip interface. Machine Science and Technology 9(1), 85-99 (2005)

3. Bachrathy, D., Stépán, G., Turi, J.: State dependent regenerative effect in milling processes. Journal of Computational and Nonlinear Dynamics 6(4), 041002 (9 pages) (2011)

4. Bagchi, A., Wright, P.K.: Stress analysis in machining with the use of sapphire tools. Proceedings of the Royal Society of London, Series A, Mathematical and Physical Sciences 409(1836), 99-113 (1987)

5. Barrow, G., Graham, W., Kurimoto, T., Leong, Y.F.: Determination of rake face stress distribution in orthogonal machining. International Journal of Machine Tool Design and Research 22(1), 75-85 (1982)

6. Bélair, J., Mackey, M.C.: Consumer memory and price fluctuations in commodity markets: an integrodifferential model. Journal of Dynamics and Differential Equations 1(3), 299-325 (1989)

7. Buryta, D., Sowerby, R., Yellowley, I.: Stress distributions on the rake face during orthogonal machining. International Journal of Machine Tools and Manufacture 34(5), 721-739 (1994)

8. Chandrasekaran, H., Kapoor, D.V.: Photoelastic analysis of chip-tool interface stresses. ASME Journal of Engineering for Industry 87, 495-502 (1965)

9. Childs, T.H.C., Mahdi, M.I.: On the stress distribution between the chip and tool during metal turning. CIRP Annals - Manufacturing Technology 38(1), 55-58 (1989)

10. Cooke, K.L., Huang, W.: On the problem of linearization for state-dependent delay differential equations. Proceedings of the American Mathematical Society 124(5), 1417-1426 (1996)

11. Dombóvári, Z., Stépán, G.: The effect of helix angle variation on milling stability. Journal of Manufacturning Science and Engineering - Transactions of the ASME 134(5), 051015 (6 pages) (2012)

12. Dombóvári, Z., Wilson, R.E., Stépán, G.: Estimates of the bistable region in metal cutting. Proceedings of the Royal Society A - Mathematical, Physical and Engineering Sciences 464, 3255-3271 (2008)

13. Driver, R.D.: A two-body problem of classical electrodynamics: the one-dimensional case. Annals of Physics 21(1), 122-142 (1963)

14. Engelborghs, K., Luzyanina, T., Roose, D.: Numerical bifurcation analysis of delay differential equations using DDE-BIFTOOL. ACM Transactions on Mathematical Software 28(1), 1-21 (2002)
15. Germay, C., Denoël, V., Detournay, E.: Multiple mode analysis of the self-excited vibrations of rotary drilling systems. Journal of Sound and Vibration 325(1-2), 362381 (2009)

16. Hajdu, D., Insperger, T.: Demonstration of the sensitivity of the Smith predictor to parameter uncertainties using stability diagrams. International Journal of Dynamics and Control (2014). Published online, DOI 10.1007/s40435-014-0142-1

17. Hartung, F.: Linearized stability in periodic functional differential equations with state-dependent delays. Journal of Computational and Applied Mathematics 174(2), 201-211 (2005)

18. Hartung, F., Krisztin, T., Walther, H.O., Wu, J.: Functional differential equations with state-dependent delays: theory and applications. In: P. Drábek, A. Cañada, A. Fonda (eds.) Handbook of Differential Equations: Ordinary Differential Equations, pp. 435-545. Elsevier/North-Holland, Amsterdam (2006)

19. Hartung, F., Turi, J.: Stability in a class of functional differential equations with state-dependent delays. In: C. Corduneanu (ed.) Qualitative problems for differential equations and control theory, pp. 15-31. World Scientific, Singapore (1995)

20. Hartung, F., Turi, J.: On differentiability of solutions with respect to parameters in state-dependent delay equations. Journal of Differential Equations 135(2), 192237 (1997)

21. Hartung, F., Turi, J.: Linearized stability in functionaldifferential equations with state-dependent delays. In: Proceedings of the conference Dynamical Systems and Differential Equations, added volume of Discrete and Continuous Dynamical Systems, pp. 416-425 (2000)

22. Insperger, T., Barton, D.A.W., Stépán, G.: Criticality of hopf bifurcation in state-dependent delay model of turning processes. International Journal of Non-Linear Mechanics 43(2), 140-149 (2008)

23. Insperger, T., Stépán, G., Turi, J.: State-dependent delay in regenerative turning processes. Nonlinear Dynamics 47(1-3), 275-283 (2007)

24. Kato, S., Yamaguchi, K., Yamada, M.: Stress distribution at the interface between tool and chip in machining. ASME Journal of Engineering for Industry 94(2), 683$689(1972)$

25. Kilic, D.S., Raman, S.: Observations of the tool-chip boundary conditions in turning of aluminum alloys. Wear 262(7-8), 889-904 (2007)

26. Kuang, Y.: Delay differential equations with applications in population dynamics. Academic Press, New York (1993)

27. Lehotzky, D., Turi, J., Insperger, T.: Stabilizability diagram for turning processes subjected to digital PD control. International Journal of Dynamics and Control 2(1), 46-54 (2014)

28. Liu, X., Vlajic, N., Long, X., Meng, G., Balachandran, B.: Nonlinear motions of a flexible rotor with a drill bit: stick-slip and delay effects. Nonlinear Dynamics 72(1-2), 61-77 (2013)

29. Liu, X., Vlajic, N., Long, X., Meng, G., Balachandran, B.: State-dependent delay influenced drill-string oscillations and stability analysis. Journal of Vibration and Acoustics 136(5), 051008 (9 pages) (2014)

30. Manitius, A.Z., Olbrot, A.W.: Finite spectrum assignment problem for systems with delays. IEEE Transactions on Automatic Control AC-24(4), 541-553 (1979)

31. Molnár, T.G., Insperger, T.: On the robust stabilizability of unstable systems with feedback delay 
by finite spectrum assignment. Journal of Vibration and Control (2014). Published online, DOI: $10.1177 / 1077546314529602$

32. Molnár, T.G., Insperger, T.: On the effect of distributed regenerative delay on the stability lobe diagrams of milling processes. Periodica Polytechnica - Mechanical Engineering 59(3), 126-136 (2015)

33. Molnár., T.G., Insperger, T., Hogan., S.J., Stépán, G.: Investigating multiscale phenomena in machining: the effect of cutting-force distribution along the tool's rake face on process stability. In: Proceedings of the ASME International Design Engineering Technical Conferences, DETC2015-47165. Boston, MA (2015)

34. Sieber, J., Engelborghs, K., Luzyanina, T., Samaey, G., Roose, D.: DDE-BIFTOOL v. 3.0 Manual Bifurcation analysis of delay differential equations (2014). URL http://arxiv.org/abs/1406.7144. Http://arxiv.org/abs/1406.7144

35. Smith, H.: An Introduction to Delay Differential Equations with Applications to the Life Sciences. Springer, New York (2011)

36. Stépán, G.: Delay-differential equation models for machine tool chatter. In: F.C. Moon (ed.) Dynamics and Chaos in Manufacturing Processes, pp. 165-192. John Wiley and Sons, New York (1997)

37. Stépán, G.: Delay, nonlinear oscillations and shimmying wheels. In: F.C. Moon (ed.) New Applications of Nonlinear and Chaotic Dynamics in Mechanics, pp. 373-386. Kluwer Academic Publisher, Dordrecht (1999)

38. Stépán, G., Kalmár-Nagy, T.: Nonlinear regenerative machine tool vibrations. In: Proceedings of DETC'97, ASME Design and Technical Conferences, pp. 1-11. Sacramento, CA, USA (1997)

39. Sutter, G., Molinari, A., List, G., Bi, X.: Chip flow and scaling laws in high speed metal cutting. ASME Journal of Manufacturing Science and Engineering 134(2), 021005 (9 pages) (2012)

40. Szalai, R., Orosz, G.: Decomposing the dynamics of heterogeneous delayed networks with applications to connected vehicle systems. Physical Review 88(4), 040902 (2013)

41. Takács, D., Orosz., G., Stépán, G.: Delay effects in shimmy dynamics of wheels with stretched string-like tyres. European Journal of Mechanics A/Solids 28(3), 516-525 (2009)

42. Toropov, A., Ko, S.L.: Prediction of tool-chip contact length using a new slip-line solution for orthogonal cutting. International Journal of Machine Tools and Manufacture 43(12), 1209-1215 (2003)

43. Usui, E., Takeyama, H.: A photoelastic analysis of machining stresses. ASME Journal of Engineering for Industry 82, 303-307 (1960)

44. Woon, K.S., Rahman, M., Neo, K.S., Liu, K.: The effect of tool edge radius on the contact phenomenon of toolbased micromachining. International Journal of Machine Tools and Manufacture 48(12-13), 1395-1407 (2008)

45. Yang, X., Liu, C.R.: A new stress-based model of friction behavior in machining and its significant impact on residual stresses computed by finite element method. International Journal of Mechanical Sciences 44(4), 703-723 (2002) 